\title{
Decimal Sequence based Imperceptible Audio Watermarking
}

\author{
Chanchal Ghosh \\ Assistant Professor \\ Calcutta Institute Of technology \\ Department Of MCA
}

\author{
Angshuman Majumdar \\ Assistant Professor \\ Calcutta Institute Of technology \\ Department Of ECE
}

\begin{abstract}
Digital watermarking has been proposed as a new, alternative method to enforce intellectual property rights and protect digital media from tempering. Digital watermarking is defined as imperceptible, robust and secure communication of data related to the host signal, which includes embedding into and extracted from the host signal. The main challenge in digital audio watermarking is that if the perceptual transmitter is fixed, the design of watermark system cannot obtain high watermark data rate and high robustness at the same time.
\end{abstract}

The main objective of this paper is to development of an audio watermarking algorithm which obtain high watermark data rate and high quality of audio at the same time

\section{General Terms}

Decimal Sequence, Audio Watermarking.

\section{Keywords}

Decimal Sequence, DCT, IDCT

\section{INTRODUCTION}

Now internet is a very good medium for distribution of digital media because of its inexpensiveness and efficiency. Also the digital data can be readily shares, easily used, processed and transmitted which causeserious problems such as unauthorized used and manipulation of digital media. As a result of this we need authentication techniques to secure the transmitted digital media. Digital watermarking is such a technique which embeds additional information with digital media to secure it .Watermark is a hidden image added to an audio that can be detected or extracted later for proof of authentication

\subsection{Application of Watermarking [1]}

The digital watermarking is use for various purposes. A few most common applications involve.

\subsubsection{Owner Identification.}

The owner identification can be printed on the cover. Thistypes of identification can be easily removed by cropping the image or by tearing the part of that has identification. Digital watermarking helps to overcome the problem by embedding the watermark in the bit position of the content. When the device read the watermark identifies the owner of the content.

\subsubsection{Copy Protection.}

The digital media can easily copy. To prevent the data from the illegal copy we used watermarking. A watermark can be done in the position of copy protection bit. When the copying device reads the data the watermark detectand stop copying.This would need all the copying machines to have the watermark circuitry to identify the watermark and act accordingly [1].

\subsubsection{Broadcast monitoring.}

A commercial advertisement may be watermarked by putting a unique watermark in each video or sound clip prior to broadcast. Automated monitoring systems can then receive broadcasts and check for these watermarks, identifying when and where each clip appears. This proves very helpful for the advertisers as they actually pay for only the number of times the advertisement was actually relayed [7].

\subsubsection{Medical applications.}

Names of the patients can be printed on the X-ray reports and MRI scans using techniques of visible watermarking. The medical reports play a very important role in the treatment offered to the patient. If there is a mix up in the reports of two patients this could lead to a disaster.

\subsubsection{Data protection.}

A given set of data (audio) can be easily tampered without even being detected. To avoid this and maintain the originality of the audio a watermark like signature, a set of words, may be embedded into the audio. If the audio is now being tampered it can be easily detected as the bit values of the embedded data would change and not match the original bit values. If the audio is being copied it would lose its authentication as the embedded data would not be copied along with the audio.

\subsection{Requirements for Good Audio Watermarking}

\subsubsection{Robustness.}

Robustness means that the watermarking scheme employed should be able to preserve the watermark under various attacks. The attack could be anything like Add noise, Add echo, Amplitude modification, resampling, Low pass filtering, Mp3 compression, Time stretch, cut samples. There might be some noise introduced by this processing but this should not affect the retrieval of the watermark.

\subsubsection{Quality of Audio orImperceptibility of Audio.}

Watermarking should be done in a way such that it does not affect the quality of the audio or the hidden data after watermarking. The changes in the audio should not be noticeable to the normal ear. 


\subsubsection{Payload capacity of the audio.}

It is very important to find the maximum amount of information that can be safely hidden in a audio. Various applications have different sizes of the data that is to be hidden. This directly affects the robustness and the perceptual impact. If too much of the data is hidden in the audio (much more than the payload capacity) it is harmful for the quality of audio as the resolution of the images reduces drastically.

\subsubsection{Reliability of the watermark.}

There is always a possibility that the user knows the exact algorithm for detecting and rendering the watermark inactive. The only way to secure the watermark then lies in the selection of the key used for watermarking. Now, even if the user on the other side knows the exact algorithm .it should be practically impossible to find the exact key to match with the one during embedding. This counts for the reliability or strength of the watermark.

\section{CONCEPT OF DECIMAL SEQUENCE}

\section{$[16,17,20]$}

Decimal sequences are obtained when a number is represented in a decimal form in a base $r$ and they may terminate, repeat or be aperiodic. As these sequences are periodic their randomness needs to be checked only in one period. For a certain class of decimal sequences of $1 / \mathrm{q}$, q prime, the digits spaced half a period apart add up to $r-1$, where $r$ is the base in which the sequence is expressed. These properties of decimal sequences have made it possible to establish an upper bound on the autocorrelation function. Decimal sequences are known to have good cross- and auto-correlation properties and they can be used in applications involving pseudorandom sequences [16] [17]. The following section describes the properties of decimal sequences.

\subsection{Properties of Decimal sequence.}

Any positive number as a decimal in the base $r$ can be represented as

$$
A 1 A 2 \ldots \ldots \ldots \ldots \ldots \text { As }+1 . a 1 a 2 \ldots \ldots \ldots \ldots \ldots
$$

Where $0 \leq A i \leq r, 0 \leq a i \leq r$, not all $\mathrm{A}$ and a are zero, and aninfinity of the $\mathrm{a}_{\mathrm{i}}$ are lessthan (r-1). There exists a one to one correspondence between the numbers and thedecimals, and $\mathrm{x}=\mathrm{A}_{1} \mathrm{r}^{\mathrm{s}}+\mathrm{A}_{2} \mathrm{r}^{\mathrm{s}-1}+\ldots . .+\mathrm{A}_{\mathrm{s}+1}+\mathrm{a}_{1} / \mathrm{r}+\mathrm{a}_{1} / \mathrm{r}^{2}+\ldots$

Decimal sequences can be used for rational and irrational numbers to generatepseudorandom noise sequences. Following are some theorems on decimal sequences

Theorem 1: If $q$ is prime and $r$ is a primitive root of $q$, then the decimal sequence for $1 / q$ is termed as maximal length decimal sequence in the base $r$.

Theorem 2: A maximal length decimal sequence $\{1 / \mathrm{q}\}$, when multiplied by $p, p<q$, is acyclic permutation of itself.

Theorem 3: If the decimal sequence in base $r$ of $p / q ;(p, q)=$ $1, p<q$, and $(r, p)=1$ isshifted to the left in a cyclic manner 1 times, the resulting sequence corresponds to thenumber $\mathrm{p} / \mathrm{q}$, $\left(p^{\prime}, q\right)=1$, p $^{\prime}<$ q where p' $\equiv$ r' X p (modulo q) $^{\prime}$

Theorem 4: For a maximum length sequence

$(1 / \mathrm{q})=\mathrm{a}_{1} \mathrm{a}_{2} \ldots \ldots \ldots \mathrm{a}_{\mathrm{k}}, \mathrm{k}=\mathrm{q}-1$ in base $\mathrm{r}$ :

$$
\mathrm{a}_{\mathrm{i}}+\mathrm{a}_{(\mathrm{k} / 2+\mathrm{i})}=\mathrm{r}-1
$$

Example: let $x=\{1 / 17\}$ in base 10

The Decimal sequence for $\mathrm{x}$ is 0588235294117647 Note that $\mathrm{a}_{\mathrm{i}}+\mathrm{a}_{8+\mathrm{i}}=\mathrm{r}-1=9$

\subsubsection{Autocorrelation Property}

For a symmetric binary decimal sequence, the autocorrelation $\operatorname{Rx}(\mathrm{j}) \leq 1-2 / m, j \neq 0, j<k$. Thus a lower bound exists on the distance between a sequence and its cyclicshifts. For a normal number, the autocorrelation function is defined as
$\mathrm{R}(\mathrm{t})=\mathrm{E}\left(\mathrm{a}_{\mathrm{n}}, \mathrm{a}_{\mathrm{n}}+\mathrm{t}\right)$

where the $\mathrm{n}^{\text {th }}$ digit of the sequence $a_{n} \in\{0,1,2, \ldots, r-1\}$. Since each of the digits occurwith a frequency $1 / \mathrm{r}, \mathrm{Rx}(0)=\mathrm{E}\left(\mathrm{a}_{\mathrm{n}}{ }^{2}\right)=$ $(\mathrm{r}-1)(2 \mathrm{r}-1) / 6$. Also for such a number, the

successive sequence of digits are independent and therefore $\operatorname{Rx}(\mathrm{t})=\mathrm{E}\left(\mathrm{a}_{\mathrm{n}}, \mathrm{a}_{\mathrm{n}+\mathrm{t}}\right)=\mathrm{E}\left(\mathrm{a}_{\mathrm{n}}\right) \mathrm{E}\left(\mathrm{a}_{\mathrm{n}+\mathrm{t}}\right)=(\mathrm{r}-1)^{2} / 4$

The autocorrelation function is two valued if the digits from zero to ( $\mathrm{r}-1)$ aremapped symmetrically about zero by the transformation $\mathrm{a}_{\mathrm{i}}{ }^{\prime}=2 \mathrm{a}_{\mathrm{i}}-(\mathrm{r}-1)$. Astraightforward calculation shows that

$\operatorname{Rx}(\mathrm{t})=\stackrel{\left(\mathrm{r}^{2}-1\right)}{\stackrel{t=0 ;}{ }} \begin{array}{ll} \\ 0 & \text { otherwise }\end{array}$

\subsubsection{Cross-Correlation Property}

Let $\mathrm{R}_{\mathrm{xy}}(\mathrm{t})=1 / \mathrm{N} \sum \mathrm{a}_{\mathrm{i}} \mathrm{b}_{\mathrm{i}+\mathrm{t}}$ represent the cross-correlation function of two maximallength sequences $\{\mathrm{x}\}=\mathrm{a} 1 \mathrm{a} 2 \ldots \mathrm{ak} 1$ and $\{\mathrm{y}\}=\mathrm{b} 1 \mathrm{~b} 2 \ldots . . \mathrm{bk} 2$. The period of the product sequenceaibi+t is $\mathrm{N}=\mathrm{LCM}(\mathrm{k} 1, \mathrm{k} 2)$.This property may be useful for the part of security against unauthorized detection of the watermark by unauthorized users who don't know the actual decimal sequence.

Theorem 5: The cross-correlation function of two maximal length sequences in thesymmetric form is identically equal to zero if the ratio $\mathrm{k} 1 / \mathrm{k} 2$ of their periods reduces to anirreducible fraction $\mathrm{n} 1 / \mathrm{n} 2$ where either $\mathrm{n} 1$ or $\mathrm{n} 2$ is and even number.

\subsection{Generating the Decimal Sequences}

A decimal sequence is generated in Matlab using the function

$$
d s e q=\left[r^{i} \bmod q\right] \bmod r
$$

Where, $\mathrm{r}$ is the radix or the base and $\mathrm{q}$ is the prime number. There is a tradeoff between the robustness and the quality of the audio as the gain $\mathrm{K}$ is increased. But, the decimal sequences gives us an option of experimenting with various prime numbers, keeping the gain constant, until we observe a satisfactory result for both encryption of data and its retrieval. For retrieval of the encrypted message the decimal sequences are generated again and then correlated with the watermarked audio.

\section{CONCEPT OF DISCRETE COSINE TRANSFORMATION (DCT) $[8,9,10]$}

The discrete cosine transform (DCT) is a technique for converting a signal into elementary frequency components. The DCT does a better job of concentrating energy into lower order coefficients than does the DFT for audio data. The DCT is purely real; the DFT is complex (magnitude and phase). Discrete Cosine Transform is a Fourier-related transform similar to the discrete Fourier transform (DFT), but using only real numbers. It is equivalent to a DFT of roughly twice the length, operating on real data with even symmetry (since the Fourier transform of a real and even function is real and even), where in some variants the input and/or output data are shifted by half a sample. (There are eight standard variants, of which four are common.). Similar to discrete Fourier transform (DFT), discrete cosine transform (DCT) is a function that maps the input signal or image from spatial domain to frequency domain. DCT transforms the input into a linear combination of weighted basis functions. These basis functions are the frequency component of the input data. An $\mathrm{N}$-point DCT has the same frequency resolution as and is closely related to a $2 \mathrm{~N}$ - point DFT. The $\mathrm{N}$ frequencies of a $2 \mathrm{~N}$ point DFT correspond to $\mathrm{N}$ points on the upper half of the unit 
circle in the complex frequency plane. The formula for DCT is

$y(k)=\sum_{n=1}^{N}\left(x(n) \cos \frac{\pi(2 n-1)(k-1)}{1 N}\right) k=1,2, \ldots . N$

Where

$$
w(k)= \begin{cases}\frac{1}{\sqrt{N}}, & k=1 \\ \sqrt{\frac{2}{N}}, & 2 \leq k \leq N\end{cases}
$$

The formula for IDCT is

$$
\begin{gathered}
x(n)=\sum_{n=1}^{N}\left(w(k) y(k) \cos \frac{\pi(2 n-1)(k-1)}{1 N}\right) \\
k=1,2, \ldots . . N
\end{gathered}
$$

Where

$$
w(k)= \begin{cases}\frac{1}{\sqrt{N}}, & k=1 \\ \sqrt{\frac{2}{N}}, & 2 \leq k \leq N\end{cases}
$$

If we apply the DCT in a audio signal then the signal becomes $2 \mathrm{~N}$ in length and duplicated its value in reverse order as shown in Fig1.

IF
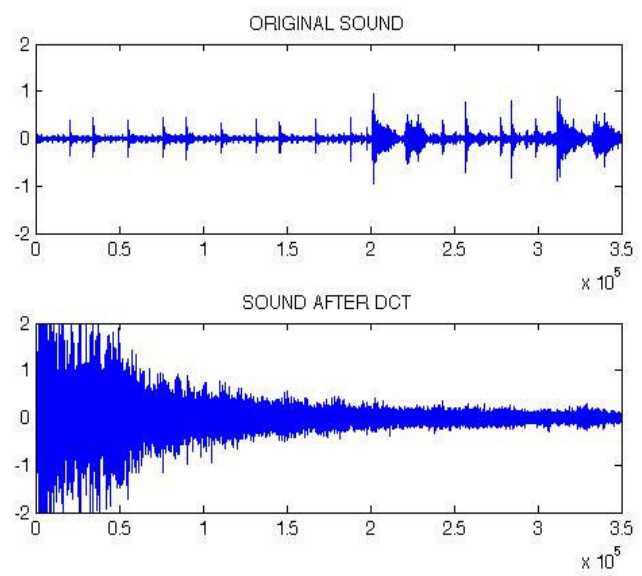

Fig1: Conversion of audio signal From Time Domain to Frequency Domain using DCT.

\section{PROPOSED ALGORITHM}

\subsection{Algorithm for Watermark Embedding.}

Step1. Give the Authentication Key in Decimal.

Step2. Convert the Authentication Key in 8 bit binary number.
Step3. Convert the Size of Image in binary.

Step4. Convert the .bmp image into lxn matrix.

Step5. Make Watermark Data by combining the output fromin same sequence.

Step6. Make a decimal sequence of order lxn .

Step7. Spread the Watermark data into decimalsequence.Step8. From the original audio select the bit valuewherethe Watermark data should be inserted.

Step9. Make DCT of selected bit value.

Step10. Embed the watermark data into bit valueusing the formula

$$
I w(x, y)=I(x, y) *(1+k * W(x, y))
$$

where $\operatorname{Iw}(x, y)$ denotes the watermarked audio.

$I(x, y)$ denotes the actual audio.

$W(x, y)$ denotes the decimal sequence that is added tothe audio.

$$
k \text { denotes the gain factor. }
$$

Step11. Make IDCT of watermarked bit.

Step1 2. Restore bit value into the original audio signal.

Step13. Write as a audio file.

\subsection{Algorithm For Watermark Detection.}

Step1. Take the original audio file and select the bit. Then make DCT of the bit.

Step2. Take the Watermarked audio file and select the bit. Then make DCT of the bit.

Step3. Select the watermarked value bv the formula

$$
W(x, y)=\left(\frac{I w(x, y)}{I(x, y)}-1\right) / k
$$

Where, Iw $(x, y)$ denotes the watermarked Audio.

$I(x, y)$ denotes the actual Audio.

$W(x, y)$ denotes a decimal sequence that is added to the Audio.

$k$ denotes the gain factor.

Step4. Correlated with Threshold $(\mathrm{t})=$ Mean (Correlation) and make decisions if

$$
\begin{aligned}
& \text { value }<t \quad W(x, y)=1 \\
& \text { Otherwise } W(x, y)=0
\end{aligned}
$$

Step7. Detect the size of the image and make it decimal. Step8. Detect the $1 \times \mathrm{N}$ image value and make it decimal. Step9. Reshape the $1 \times \mathrm{N}$ matrix into $\mathrm{M} \times \mathrm{N}$ matrix. Step10. Write as an image file. 


\section{FLOWCHART FOR PROPOSED ALGORITHM}

\subsection{Flowchart for Watermark Embedding.}

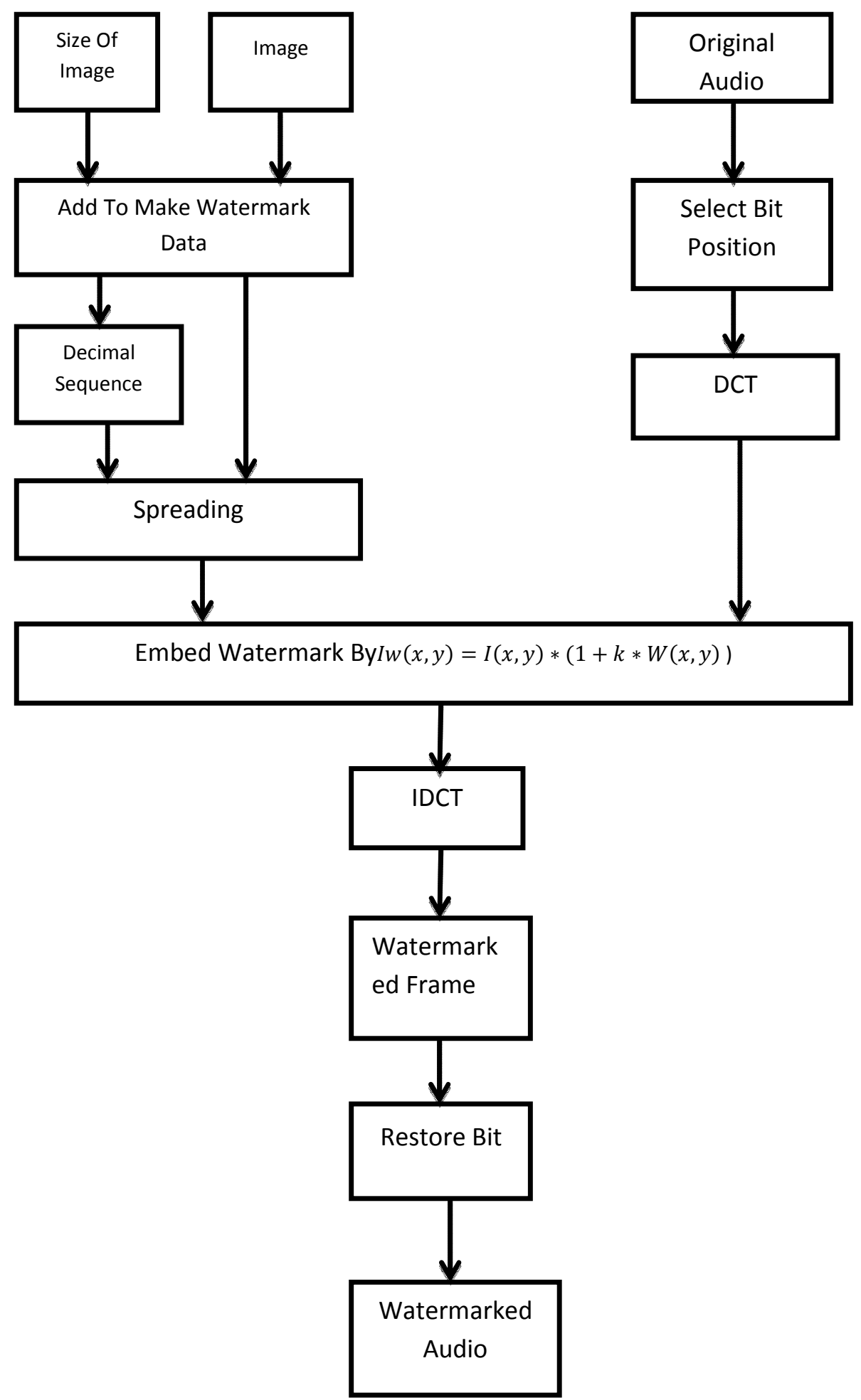

Fig 2: Flowchart for watermark embedding. 


\subsection{Flowchart for Watermark Detection}

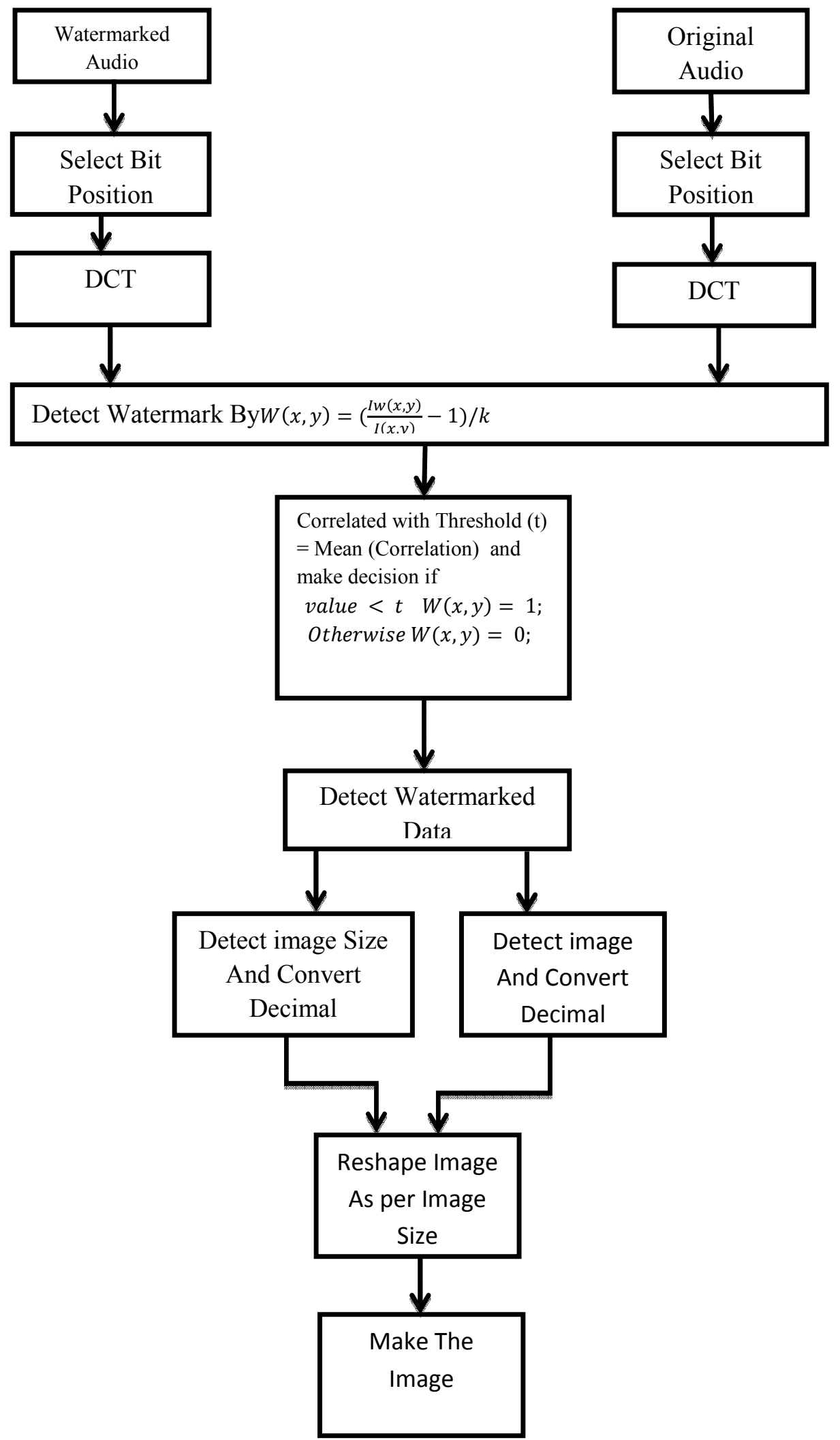

Fig3: Flowchart for Watermark Detection 


\section{EXPERIMENTAL SETUP}

To implement our proposed algorithm we used Matlab 6.5 and used an instrumental Audio, one Music Song and one Speech Audio as Cover Audio of duration $30 \mathrm{sec}, 40 \mathrm{sec}$ and $80 \mathrm{sec}$ .For watermark data we used Black and white image given below.

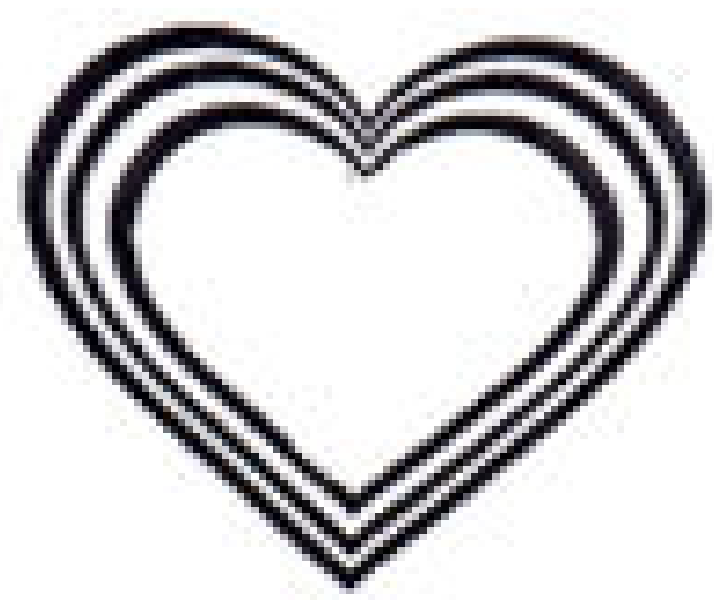

Fig4: Image used as watermarked data.

We evaluate the performance of our watermarking system for three different types of 16 bit mono audio signals sampled at $44.1 \mathrm{kHz}$ :

a)Reciting by lopamudra Mitra;

b)An instrumental song from film 'Taal';

c)An instrumental Sanai By 'Ustad BismillahKhan'.

\section{PERFORMANCE PARAMETERS}

a) In order to evaluate the quality of watermarked signal, the following signal-to-noise ratio (SNR) equation is used:

$$
S N R=10 \log _{10}\left(\frac{\sum_{a=1}^{M} Z^{2}(a)}{\sum_{a=1}^{M}\left(Z(a)-Z^{\prime}(a)\right)}\right)
$$

Where $Z(a)$ is Original audio signal.

$Z^{\prime}(a)$ is Watermarked audio signal.

$\mathrm{M}$ is the number of sample in both audio signal.

b) In order to evaluate the quality of the extracted image we used BER(bit-error-ratio) and NC( Normalize Correlation) .

The equation for BER and $\mathrm{NC}$ is given below

$$
B E R=\frac{B r r}{M \times N} \times 100 \%
$$

Where Brr is the number of error bit and $\mathrm{M} \times \mathrm{N}$ is the size of the image.

$N C$

$$
=\frac{\sum_{i=1}^{M} \sum_{j=1}^{N} Y(I, J) Y^{\prime}(i, j)}{\sqrt{\sum_{i=1}^{M} \sum_{j=1}^{N} Y(i, j)^{2} \sqrt{\sum_{i=1}^{M} \sum_{j=1}^{N} Y^{\prime}(i, j)^{2}}}}
$$

Where $Y(i, j)$ is bit value of original image and $Y^{\prime}(i, j)$ is the bit value of recovered image.

\section{RESULT}

When we apply our proposed embedding algorithm using the above mention audio signal and image we get resultant audio signal as follow
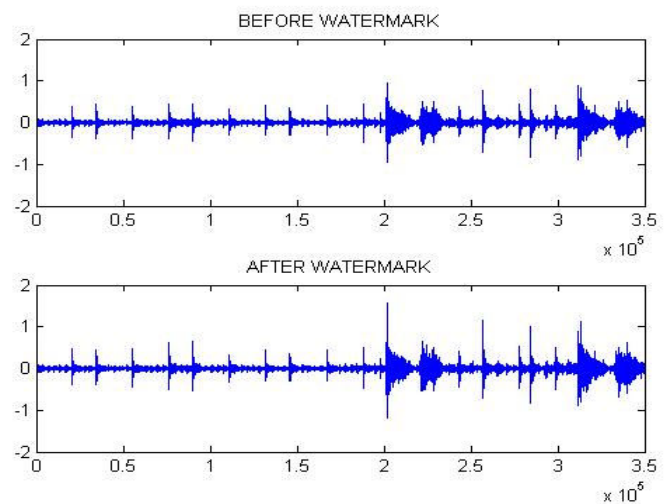

Fig5. Audio signal after watermark embedding.

When we apply our detection algorithm for watermark detection we get the watermark image as follow
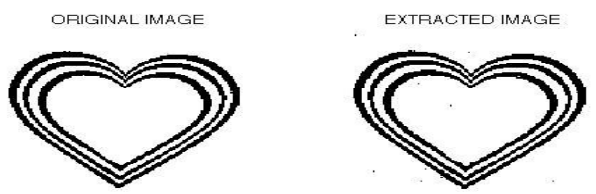

Fig6. Image after Watermark detection.

\section{PERFORMANCE ANALYSIS}

The performance of the proposed algorithm against the signal processing is evaluated. The watermark taken is a size binary image $114 \times 131$.From the observation, for SNR value of near about $21 \mathrm{~dB}$ the embedded watermark is audible to the human ear. In addition to that, the NC and BER of the extracted watermark is nearly 1 and 0 .

Table1. Performance evaluation of the embedded watermark

\begin{tabular}{|c|c|c|c|}
\hline Types of Signal & SNR & BER & NC \\
\hline $\begin{array}{c}\text { Reciting by } \\
\text { lopamudra Mitra }\end{array}$ & 21.2207 & 0.0647 & 1 \\
\hline $\begin{array}{c}\text { An instrumental } \\
\text { song from film 'Taal' }\end{array}$ & 23.4658 & 0.0449 & 1 \\
\hline $\begin{array}{c}\text { An instrumental } \\
\text { Sanai By 'Ustad } \\
\text { Bismillah Khan }\end{array}$ & 27.4767 & 0.0829 & 1 \\
\hline
\end{tabular}


Table 1 shows the SNR results of the proposed watermarking systemachieves SNR values ranging from $20 \mathrm{~dB}$ to $40 \mathrm{~dB}$ for different watermarked sounds. According to the International Federation Of The Phonographic Industry(IPPI) the SNR of the watermarked audio signal should be greater than $20 \mathrm{~dB}[22,24]$, Our proposed system achieves SNR values ranging from $20 \mathrm{~dB}$ to $30 \mathrm{~dB}$ for different watermarked sounds. The BER of our proposed system near to zero and the $\mathrm{NC}$ is one for different types of audio signal.

\section{CONCLUSION}

In this paper, we have presented a new watermarking system using discrete Cosine Transform (DCT) and decimal sequence for Watermarking of audio. Experimental results indicate that our proposed watermarking system shows strong Imperceptibility for various kind of sound. Our proposed system achieves SNR values ranging from $20 \mathrm{~dB}$ to $28 \mathrm{~dB}$ and $\mathrm{BER}$ near to zero and $\mathrm{NC}$ always one for different watermarked sounds. These results demonstrate that our proposed watermarking system can be a suitable candidate for audio Watermarking.

\section{FUTURE SCOPE}

1) We did not consider any kind of attack in watermarked audio. Our future work is to apply various kinds of attack in watermarked audio and then detection.

2) This theorem applied only for 16 bit mono sound. Our future Work is to apply this theorem for Stereo sound

3) This theorem applied for Black and White image. Our next work is to apply this theorem for colored image.

\section{REFERENCES}

[1] Katzenbeisser, S., Petitcolas, F.A.P., Information hiding techniques for steganography and digital watermarking, Artech House Publishers, 2000.

[2] Gnanaguruparan, M., "Recursive secret sharing in visual cryptography", MS thesis, Louisiana State University.

[3] Shoemaker, C., "Hidden bits: A survey of techniques for digital watermarking", Independent study, EER 290, spring 2002.

[4] Johnson, N.F., Jajodia, S., and Duric, Z., Information hiding: Steganography and watermarking attacks and countermeasures, Kluwer academic Publishers, 2000.

[5] Kutter, M., and Hartung, F., Introduction to watermarking techniques - Information technology for steganography and digital watermarking, Artec House, 2000 .

[6] Swanson M. D,, Zhu B., Tewfik A. H. and L.Boney L., "Robust Audio Watermarking Using Perceptual Masking", Elsevier SignalProcessing, Sp. Issue on Copyrights Protection and Access Control 1998 , Vol. 66, No. 3, pp. 337-355.

[7] Cox I. J., Kilian J. Leighton F. T. and Shamoon T., " Secure Spread Spectrum Watermarking for Multimedia".
IEEE Trans. On Image Processing 1997, Vol. 6, No. 12, pp. 1673- 1687

[8] Xiang-Yang Wang and Hong Zhao.," A Novel Synchronization Invariant Audio Watermarking Scheme Based on DWT and DCT". IEEE TRANSACTIONS ON SIGNAL PROCESSING Dec 2006, VOL. 54, NO. 12,pp. 4835-4840.

[9] Xiumei Wen, Xuejun Ding, Jianhua Li. LitingGao, Haoyue Sun.," An Audio Watermarking Algorithm Based on Fast Fourier Transform". International Conference on Information Management, Innovation Management and Industrial Engineering 2009.

[10] Yan Yang, Rong Huang, MintaoXu.," A Novel Audio Watermarking Algorithm for Copyright Protection Based on DCT Domain", SecondInternational Symposium on Electronic Commerce and Security 2009.

[11] Xian hang Tang, YameiNei, Hengli You, Thangke Yin., "Digital Audio watermark Embedding Algorithm",International Journal of information Technology 2005,vol-11 No-12.

[12] X.F Ma , T. Jaing., "The research on wavelet domain audio watermarking based on ICA",Journal For Physics:Conference Series 48(2006),pp.442-446.

[13] M ketcham ,S.Vongpradhip.," Intelligent audio watermarking using Genetic Algorithm in DWT domain",World Academy Of Science, Engineering And Technology 26,2007.

[14] Jonathm M blackledge.," Digital watermarking and selfauthentication using chirp coding.",IS AST Transactions On Electronics And Signal Processing 2007,vol-1,no-1.

[15] Xian hang Tang, YameiNei, Hengli You , Thangke Yin.," Digital Audio watermark Embedding Algorithm.",International Journal of Information Technology 2005., Vol- 11,No-12.

[16] Kak, S.C., and Chatterjee, A., "On Decimal Sequences", IEEE Transactions on Information Theory, vol. IT-27, No.5, pp. 647 - 652,1981.

[17] Kak, S.C., "Generating d-sequences", Electronics Letters, vol. 23, pp. 202-203,1987.

[18] W. N. Lie and L. C. Chang, "Robust and High-Quality Time-Domain Auciio Watermarking Based on LowFrequency Amplitude Modification," IEEE Transaction on Multimedia, vol. 8, no. 1, pp. 46-59, February, 2006.

[19] G. Zeng and Z. Qiu, "Audio Watermarking in DCT: Embedding Strategy and Algorithm," in Proceedings of 9th International Conference on Signal Processing (ICSP'09), pp. 2193-2196, 2008.

[20] NavneetMandhani and SubhashKak," watermarking using decimal sequence",Cryptologia, volume 29, January 2005 . 